\title{
Correspondence
}

\author{
Contents - Pituitary in psychosis - CBT for psychosis - Depression interventions \\ in resource-poor regions a ADHD in developing countries
}

\section{Pituitary in psychosis}

Pariante et al (2004) conclude that patients with first-episode psychosis have a larger pituitary volume and those with chronic schizophrenia a smaller pituitary volume in comparison with the controls. However, there are a number of factors that limit this conclusion.

First, normal variation in pituitary volume: a large degree of variation is observed in the morphology of anterior and posterior pituitary in healthy individuals (Fujisawa et al, 1987). Moreover, the variations may occur in the same individual if the measurements are repeated after an interval (Brooks et al, 1989). Therefore, conclusions based on a single measurement may be unreliable and at least two or three measurements should have been performed for better accuracy.

Second, effect of gender and age: men tend to have smaller pituitaries, as mentioned by Pariante $e t$ al, and the pituitary size decreases with age (Brooks et al, 1989). In the study by Pariante et al the schizophrenia group contained a significantly larger number of men and significantly older people compared with the control group. These differences could be partially responsible for the smaller pituitary size observed in chronic schizophrenia.

Third, failure to demonstrate the hyperactivity of hypothalamic-pituitary-adrenal (HPA) axis: the correlation between HPA axis and pituitary volume is purely speculative and Pariante et al did not discuss the negative studies on the subject. Katona \& Roth (1985) reported an abnormal dexamethasone suppression response in only $33 \%$ of patients (10 out of 30 ) with schizo-affective depression.

Fourth, failure to measure the adrenal gland size: adrenal gland hypertrophy has been shown to correlate with hyperactivity of the HPA axis in depression (Nemeroff et al, 1992). Pariante et al did not measure the adrenal gland size, probably as the study was not pre-planned and magnetic resonance imaging data obtained for another study were utilised. Measurement of adrenal gland size would have added more weight to the study findings.

Another comment worth mentioning is that hyperactivity of the HPA axis does not point to a specific diagnosis and occurs in a large number of conditions associated with stress. Therefore, this finding alone has a limited role in diagnosis of a particular condition.

Brooks, B. S., el Gammal, T., Allison, J. D., et al (1989) Frequency and variation of the posterior pituitary bright signal on MR images. American Journal of Neuroradiology, 10, 943-948.

Fujisawa, I., Asato, R., Nishimura, K., et al (1987) Anterior and posterior lobes of the pituitary gland: assessment by I.5 T MR imaging. Journal of Computer Assisted Tomography, II, 214-220.

Katona, C. L. \& Roth, M. (1985) The dexamethasone suppression test in schizo-affective depression. Journal of Affective Disorders, 8, 107-112.

Nemeroff, C. B., Krishnan, K. R., Reed, D., et al (1992) Adrenal gland enlargement in major depression. A computed tomographic study. Archives of General Psychiatry, 49, 384-387.

Pariante, C. M., Vassilopoulou, K., Velakoulis, D., et al (2004) Pituitary volume in psychosis. British Journal of Psychiatry, 185, 5-10.

S. Kumar Department of Neurological Sciences, Christian Medical College, Vellore, India 632004.

E-mail: drsudhirkumar@yahoo.com

Author's reply: We welcome Dr Kumar's comments, drawing attention to some of the limitations of our study (Pariante $e t$ al, 2004). Even though some of these points had already been discussed in the paper, we think it is helpful to reply to all comments.

We agree that there is a large degree of variation in the morphology of the pituitary. For example, in our sample approximately half the subjects had a 'concave' pituitary, and a third had a 'flat' pituitary. However, we have minimised the influence of morphology on the volume measurement by tracing all coronal slices where the pituitary was visualised. $\mathrm{Dr}$ Kumar also refers to the paper by Brooks et al (1989) showing intra-individual changes in the brightness of the posterior bright signal, representing vasopressin released in the posterior lobe for fluids control. We did not analyse the brightness of the posterior bright signal, as we were not interested in the regulation of fluids control in our sample.

In our study there were significant differences in age and gender between the groups. By definition, it was impossible to have one single control that was comparable to the young first-episode participants as well as to the older individuals with established schizophrenia, for both age and gender distribution. However, we used two strategies to control for these confounders: first, gender and age (and whole-brain volume) were included as covariates in the analysis; second, the results obtained from this analysis were further corroborated by conducting separate tests comparing the clinical groups (first-episode and established) with selected control groups that had similar age and gender distribution. Both strategies led to the same results, thus demonstrating that the smaller pituitary volume in patients with established schizophrenia is not due to differences in age and gender distribution.

We agree that the association between increased pituitary volume and HPA axis hyperactivity is speculative, and we clearly stated this in our paper (see Limitations, p.10). Nevertheless, over 30 years ago Sachar et al (1970) found that patients experiencing a first-episode psychosis were more likely to present with HPA abnormalities, because of the distress associated with the 'dramatic and ego-dystonic' nature of this experience. Several studies have confirmed that patients who are in the acute phase of a psychotic disorder, with florid symptoms, newly hospitalised or unmedicated, have elevated HPA axis activity as shown by raised cortisol levels (Sachar et al, 1970), non-suppression of cortisol secretion by dexamethasone in dexamethasone suppression test and in the dexamethasone/corticotropin-releasing factor (CRF) test (Herz et al, 1985; Lammers et al, 1995), and elevated levels of CRF in the cerebrospinal fluid (Banki et al, 1987). Only patients who are clinically stable and receiving treatment tend to have a normal HPA axis (Ismail et al, 1998). Indeed, 
the study by Katona \& Roth (1985), cited by Dr Kumar, also reports, in the authors' own words, 'an increased frequency of HPA axis abnormality' in schizoaffective depression.

We agree that our study would have been more complete if we had measured the adrenal gland size. Indeed, measuring hormonal levels would have been an important addition to the study. We will take this advice into consideration in our future studies.

Finally, we support Dr Kumar's view that hyperactivity of the HPA axis occurs in a large number of conditions associated with stress. Indeed, we suggested in our paper that glucocorticoid resistance could be the molecular mechanism by which stress induces HPA axis hyperactivity in patients with different mental disorders (see Discussion, p. 8). We never suggested that this biological abnormality could have any diagnostic value. However, we believe that measuring specific biological markers can give us further insight into the pathological mechanisms affecting the brains (and the bodies) of our patients.

Banki, C. M., Bissette, G., Arato, M., et al (1987) CSF corticotropin-releasing factor-like immunoreactivity in depression and schizophrenia. American Journal of Psychiatry, 144, 873-877.

Brooks, B. S., el Gammal, T., Allison, J. D., et al (1989) Frequency and variation of the posterior pituitary bright signal on MR images. American Journal of Neuroradiology, 10, 943-948.

Herz, M. I., Fava, G. A., Molnar, G., et al (1985) The dexamethasone suppression test in newly hospitalised schizophrenic patients. American Journal of Psychiatry 142, 127-129.

Ismail, K., Murray, R. M., Wheeler, M. J., et al (1998) The dexamethasone suppression test in schizophrenia. Psychological Medicine, 28, 3II-3I7.

Katona, C. L. \& Roth, M. (1985) The dexamethasone suppression test in schizo-affective depression. Journal of Affective Disorders, 8, 107-112.

Lammers, C. H., Garcia-Borreguero, D., Schmider, J., et al (1995) Combined dexamethasone/ corticotropin-releasing hormone test in patients with schizophrenia and in normal controls: Il. Biological Psychiatry, 38, 803-807.

Pariante, C. M., Vassilopoulou, K., Velakoulis, D., et a (2004) Pituitary volume in psychosis. British Journal of Psychiatry, 185, 5-10.

Sachar, E. J., Kanter, S. S., Buie, D., et al (1970) Psychoendocrinology of ego disintegration. American Journal of Psychiatry, 126, 1067-1078.

C. M. Pariante Division of Psychological Medicine, Box PO5I, Institute of Psychiatry, King's College London, I Windsor Walk, Denmark Hill, London SE5 8AF, UK. E-mail: spjucmp@iop.kcl.ac.uk

\section{CBT for psychosis}

Although the study by Tarrier et al (2004) appears to be methodologically more rigorous than the similar study of cognitivebehavioural therapy (CBT) in post-acute schizophrenia by Turkington et al (2002), I am a little confused by the authors' conclusions. After clearly demonstrating no superior effect for CBT over supportive counselling on measures of symptom reduction and relapse rates, the authors conclude their paper by stating that they 'suggest that the optimum psychosocial management of early schizophrenia would include a combination of CBT and family intervention'. Would it be rude to suggest that the authors take into account their own findings before making such a statement? It is also more than a little irritating that the authors refer to their sample as being diagnosed with 'early schizophrenia' throughout the paper. Examining the inclusion criteria for this study shows that patients were included who had schizophreniform disorders, delusional disorders and unspecified psychoses. Regarding the criterion for 'early', most clinicians would define the duration of schizophrenia from symptom onset to commencement of treatment (hence the concept of the duration of untreated psychosis). Unless the authors specify illness duration, the criterion of 'early' cannot be asserted. Thus, this appears to be a study of patients within 2 years of their first episode of nonaffective psychoses and not those with early schizophrenia per se.

Tarrier, N., Lewis, S., Haddock, G., et al (2004) Cognitive-behavioural therapy in first-episode and early schizophrenia: 18-month follow-up of a randomised controlled trial. British Journal of Psychiatry, 184, 231-239.

Turkington, D., Kingdon, D. \& Turner, T. (2002) Effectiveness of a brief cognitive-behavioural intervention in the treatment of schizophrenia. British Journal of Psychiatry, 180, 523-527.

A. J. Mitchell Brandon Mental Health Unit, Gwendolen Road, Leicester LE5 4PW, UK. E-mail: alex.mitchell@leicspart.nhs.uk

Authors' reply: Dr Mitchell professes both confusion and irritation at our report of the follow-up results of the SoCRATES study. I will attempt to clarify. When implemented with standard care, CBT has consistently been found to result in clinical benefits in terms of symptom relief compared with standard care alone. This has been found in post-acute illness, as with the reported study, and chronic phases (Pilling et al, 2002). Cognitive-behavioural therapy is a structured psychological treatment usually implemented from a manual, which makes it relatively amenable to 'roll-out'; CBT has less impact on relapse rates. Family interventions have been shown to have the benefit of significantly reducing relapse rates (Pilling et al, 2002). Thus, combining both CBT and family interventions would appear to be the most parsimonious way of capitalising on these developments to improve patient care by reducing symptoms of psychosis and reducing risk of subsequent relapse. In addition, both service users and carers have been increasingly vocal in wishing a greater range of interventions to be made available, including both psychological treatments and assistance for families. It is regrettable that Dr Mitchell's comments implicitly appear to wish to deny them these further options. With respect to his comment on our inclusion criteria for the trial, a first episode of psychosis resulting in treatment by mental health services is an event that can be identified with reasonable accuracy (at least, much more accurately than emergence of symptoms prior to this). As $80 \%$ of study participants had first-episode illness using this criterion, the use of the term 'recent onset' is not unreasonable. The inclusion of those suffering from schizophreniform psychosis, delusional disorders and unspecified psychosis reflects clinical practice and conforms to convention on large pragmatic clinical trials in having broad inclusion criteria to aid generalisation (see Johnson, 1998).

Johnson, T. (1998) Clinical trials in psychiatry: background and statistical perspective. Statistical Methods in Medical Research, 7, 209-234.

Pilling, S., Bebbington, P., Kuipers, E., et al (2002) Psychological treatments in schizophrenia: I. Metaanalysis of family interventions and cognitive behaviour therapy. Psychological Medicine, 32, 763-782.

N. Tarrier, G. Haddock, S. Lewis School of Psychiatry and Behavioural Sciences, Education and Research Centre (2nd floor), Wythenshawe Hospital, Southmoor Road, Manchester M23 9LT, UK. E-mail: nicholas.tarrier@man.ac.uk

\section{Depression intervention in resource-poor regions}

Depression is recognised as a common problem in developing countries and it is one of the most important causes of morbidity and mortality (Patel et al, 2001). Lack of 